\title{
Accurate confidence intervals for risk difference in meta-analysis with rare events
}

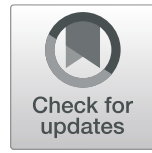

Tao Jiang ${ }^{1 *}+$, Baixin $\mathrm{CaO}^{2}$ and Guogen Shan ${ }^{3 *}$

\begin{abstract}
Background: Meta-analysis provides a useful statistical tool to effectively estimate treatment effect from multiple studies. When the outcome is binary and it is rare (e.g., safety data in clinical trials), the traditionally used methods may have unsatisfactory performance.

Methods: We propose using importance sampling to compute confidence intervals for risk difference in meta-analysis with rare events. The proposed intervals are not exact, but they often have the coverage probabilities close to the nominal level. We compare the proposed accurate intervals with the existing intervals from the fixed- or random-effects models and the interval by Tian et al. (2009).

Results: We conduct extensive simulation studies to compare them with regards to coverage probability and average length, when data are simulated under the homogeneity or heterogeneity assumption of study effects.

Conclusions: The proposed accurate interval based on the random-effects model for sample space ordering generally has satisfactory performance under the heterogeneity assumption, while the traditionally used interval based on the fixed-effects model works well when the studies are homogeneous.
\end{abstract}

Keywords: Binary outcome, Confidence interval, Importance sampling, Meta-analysis, Rare events

\section{Background}

Meta-analysis is a useful statistical tool in medical research to evaluate treatment effect by analyzing outcomes from multiple clinical trials. The estimated treatment effect from meta-analysis is always more reliable and accurate than the estimate from one selected study among the available studies. In early phase clinical trials to study safety of a new drug, rare events are very common [1]. In meta-analysis for such data, Vandermeer et al. [1] pointed out that the traditionally used asymptotic point estimates and confidence intervals could be substantially different from the results using exact methods under the

\footnotetext{
*Correspondence: jtao@263.net; guogen.shan@unlv.edu

†Tao Jiang and Guogen Shan contributed equally to this work.

'School of Statistics and Mathematics, and School of Business, Zhejiang

Gongshang University, Hangzhou, Zhejiang, China

${ }^{3}$ Epidemiology and Biostatistics Program, School of Public Health, University of Nevada Las Vegas, Las Vegas, USA

Full list of author information is available at the end of the article
}

exact conditional framework [2]. It is well known that asymptotic approaches often do not have satisfactory performance when outcome is extreme or sample size is small.

Multiple methods have been developed for metaanalysis with rare events over decades $[3,4]$. The fixedeffects models are conveniently used in practice, such as the Mantel-Haenszel method [5]. When one or both groups in a study have zero events, a continuity correction is often needed in order to estimate risk ratio or odds ratio, but the traditional correction by adding 0.5 may lead to undesirable influence on the analysis results as pointed out by Sweeting et al [6]. Later, they developed a continuity correction method by adding a float value based on the size of each group to improve the coverage probability. Multiple follow-up articles discussed this issue whether or not a small value should be added to studies with rare events in data analysis [7, 8]. Kuss et al. [8] suggested using a beta-binomial model to avoid adding 
arbitrary values to each cell in data analysis. Recently, Tian et al. [9] proposed a simple and effective method for confidence interval calculation without artificial continuity correction. The confidence intervals from each study were weighted to construct an overall interval from simulation studies under the fixed-effects model. Their developed confidence intervals were shown to have better coverages when the events are rare, but the length of their intervals could be much longer than others.

In contrast to fixed-effects models, the treatment effect in the random-effects model is assumed to follow a normal distribution. DerSimonian and Laird [10] proposed a random-effects model by including a random study effect to account for the variation of study population or study design. The statistical software $\mathrm{R}$ package meta can be used to compute confidence intervals for the fixed-effects model and random-effects model [11]. Recently, Bakbergenuly and Kulinskaya [12] suggested the generalized linear mixed models (GLMMs) in meta-analysis to include the correlation between point estimate and its variance estimate in data analysis.

The aforementioned exact conditional approach assumes both marginal totals in each study fixed [2]. It is reasonable to assume that the numbers of participant in each treatment group are fixed. It is not usual that a repeated study has the same total number of events as the observed study. The exact one-sided limit by Buehler [13] follows the study design with sample size in each treatment group fixed [14-16]. However, it is too computationally intensive to generate all possible samples in meta-analysis with binary outcome [17].

In this article, we propose using importance sampling to construct confidence interval for risk difference in metaanalysis with rare events. We apply the importance sampling method described by Lloyd and Li [18] to compute the profile confidence limit proposed by Kabaila and Lloyd [19]. Importance sampling methods have been studied by many researchers with regards to coverages of confidence intervals $[20,21]$. Importance sampling does not require to enumerate all possible samples [19]. This approach simulates samples from the distribution estimated from the observed data. Importance sampling has to be used in conjunction with a designated statistic to order the limits of simulated samples. We consider the existing intervals from the fixed-effects and random-effects models as designated statistics in this article.

The rest of this article is organized as follows. In "Methods" section, we describe the fixed-effects and random-effects models to estimate confidence intervals for risk difference. We then introduce importance sampling for interval calculation. In "Results" section, we use an example from 18 schizophrenia clinical trials to illustrate the application of the proposed intervals, and then compare the proposed intervals with the existing inte rvals with regards to coverage probability and average length. In "Conclusions" section, we provide some remarks on data analysis for meta-analysis with rare events.

\section{Methods}

For meta-analysis with binary outcome, data can be organized in a $K \times 4$ table, where $K$ is the number of studies (Table 1). Each row represents the results from a parallel study with the number of events and the number of nonevents in the new treatment group and the control group, respectively. Let the two treatment groups be indexed by 0 and 1 for the control and the new treatment, respectively. Suppose $X_{i j r}$ is the number of participants having $r$ events from the treatment $j$ in $i$ th study, where $i=1,2, \cdots, K$, $j=0,1$, and $r=0,1$. For studies with rare events, $X_{i j 1}$ is often very small. Let $n_{i j}=X_{i j 1}+X_{i j 0}$ be the total number of participants from the treatment $j$ in the $i$ th study, and $N_{1}=\left(n_{11}, n_{21}, \cdots, n_{K 1}\right)$ and $N_{0}=\left(n_{10}, n_{20}, \cdots, n_{K 0}\right)$ be the sample sizes for the new treatment group and the control group, respectively. Suppose $p_{j}$ is the event rate of the treatment $j$. Given the sample size $n_{i j}$, the number of responses among these participants, $X_{i j 1}$, follows a binomial distribution, $B\left(n_{i j}, p_{j}\right)$. We assume that each study is independent from each other, and the two groups within each study are independent from each other as well. The parameter of interest here is the risk difference between the treatment group and the control group,

$$
\Delta=p_{1}-p_{0} .
$$

We first review the existing methods to construct twosided confidence intervals for $\Delta$ in "Intervals based on fixed or random-effects model" section, and then develop accurate intervals in "Accurate intervals" section.

\section{Intervals based on fixed or random-effects model}

We first consider the fixed-effects model to calculate confidence interval for $\Delta$. Under the study homogeneity assumption, the treatment effect in each study is assumed to be the same,

$$
\Delta_{i}=\mu,
$$

where $\mu$ is the treatment effect. In the $i$ th study, the risk difference $\Delta_{i}$ is estimated as

$$
\widehat{\Delta}_{i}=\hat{p}_{i 1}-\hat{p}_{i 0},
$$

\begin{tabular}{|c|c|c|c|c|}
\hline \multirow[t]{2}{*}{ Study } & \multicolumn{2}{|c|}{ Treatment group } & \multicolumn{2}{|c|}{ Control group } \\
\hline & Events & Non-events & Events & Non-events \\
\hline 1 & $X_{111}$ & $X_{110}$ & $X_{121}$ & $X_{120}$ \\
\hline 2 & $X_{211}$ & $X_{210}$ & $X_{221}$ & $x_{220}$ \\
\hline$\cdots$ & $\cdots$ & $\cdots$ & $\cdots$ & $\cdots$ \\
\hline K & $X_{K 11}$ & $X_{K 10}$ & $X_{K 21}$ & $x_{K 20}$ \\
\hline
\end{tabular}

Table 1 Data from $K$ independent studies with binary outcome 
where $\hat{p}_{i j}=X_{i j 1} / n_{i j}$ is the estimated rate of the treatment $j$ in the $i$ th study. The variance is estimated as $s_{i}^{2}=\sum_{j=0}^{1} \frac{\hat{p}_{i j}\left(1-\hat{p}_{i j}\right)}{n_{i j}}$ from two independent proportions. The weight for the $i$ th study is

$$
w_{i}=\frac{n_{i 1} n_{i 0}}{n_{i 1}+n_{i 0}} \frac{1}{\sum_{i=1}^{K} \frac{n_{i 1} n_{i 0}}{n_{i 1}+n_{i 0}}},
$$

where $\sum_{i=1}^{K} \frac{n_{i 1} n_{i 0}}{n_{i 1}+n_{i 0}}$ is the factor to standardize the weight values, with $\sum_{i=1}^{K} w_{i}=1$. It is easy to show that $w_{i}$ is an increasing function of $n_{i 1}\left(n_{i 0}\right)$ when $n_{i 0}\left(n_{i 1}\right)$ is fixed.

The overall weighted treatment effect using the fixedeffects model is calculated as

$$
\widehat{\Delta}_{F}=\sum_{i=1}^{K} w_{i} \widehat{\Delta}_{i} .
$$

and its variance is estimated as

$$
\widehat{S E}_{F}^{2}=\sum_{i=1}^{K} w_{i}^{2} s_{i}^{2}
$$

The standardized statistic $\widehat{\Delta} / \widehat{S E}_{F}$ follows the standard normal distribution asymptotically when $\Delta=0$. Therefore, the asymptotic confidence interval for $\Delta$ based on the fixed-effects model (the $\mathrm{F}$ interval) at the nominal level of $100(1-\alpha) \%$ is

$$
C I_{F}=\left(\widehat{\Delta}_{F}-z_{1-\alpha / 2} \widehat{S E}_{F}, \widehat{\Delta}_{F}+z_{1-\alpha / 2} \widehat{S E}_{F}\right),
$$

where $z_{a}$ is the ath quantile of the standard normal distribution.

In the observation of study heterogeneity which could be caused by study population or study design or influential covariates, DerSimonian and Laird [10] proposed using the random-effects model to include the study random effect in the model as

$$
\Delta_{i}=\mu+u_{i},
$$

where $u_{i}$ is the deviation of the $i$ th study from the population mean $\mu$, and it follows a normal distribution. Let $v_{i}$ be the weight of the $i$ th study from the fitted random-effects model. Then, the weighted treatment effect and its variance are $\widehat{\triangle}_{R}=\sum_{i=1}^{K} v_{i} \widehat{\Delta}_{i}$, and $\widehat{S E}_{R}^{2}=\sum_{i=1}^{K} v_{i}^{2} s_{i}^{2}$, respectively. It follows that the asymptotic confidence interval for $\Delta$ using the random-effects model (the $\mathrm{R}$ interval) is computed as

$$
C I_{R}=\left(\widehat{\Delta}_{R}-z_{1-\alpha / 2} \widehat{S E}_{R}, \widehat{\Delta}_{R}+z_{1-\alpha / 2} \widehat{S E}_{R}\right),
$$

It can be seen that the difference between $C I_{F}$ and $C I_{R}$ is the weights used in the treatment effect and its variance calculation. The $\mathrm{F}$ interval and the $\mathrm{R}$ interval can be computed by using the function metabin from the statistical software package meta $[11,22]$. In the metabin function, we use $M H$.exact $=$ TRUE in the option with no continuity correction in the estimates.

\section{Accurate intervals}

Exact confidence limit by Buehler [13] for $\Delta$ is preferable, but it is computationally intensive to save all the possible samples in meta-analysis with sample size $n_{i j}$ fixed. For this reason, we consider importance sampling (IS) to construct accurate intervals for $\Delta$ by simulating samples from the distribution estimated from the observed data to make statistical inference. Importance sampling has been applied to many important medical research areas that often only have one nuisance parameter (e.g., the proportion difference in a parallel study [21, 23]). We extend the application of IS to meta-analysis with multiple nuisance parameters in confidence interval calculation. The intervals computed using importance sampling are accurate with coverage close to the nominal level. In addition, importance sampling has the computational advantage over exact methods [19].

The calculation of the IS intervals has to be used in conjunction with a designated statistic for the interval ordering. Let $T$ be the considered designated statistic. Suppose $\mathbf{p}_{0}=\left(p_{10}, p_{20}, \cdots, p_{K 0}\right)$ is the probability vector of the control group, where $p_{i 0}$ is the probability of the control group in the $i$ th study. The accurate upper limit based on the designated statistic $T$ is computed as the supremum of $\Delta$ such that

$$
G(\Delta)=P\left(T(\mathbf{Y}) \leq T\left(\mathbf{y}_{\mathbf{o b s}}\right) \mid \Delta, \hat{\mathbf{p}}_{0}(\Delta)\right)>\frac{\alpha}{2},
$$

where $\mathbf{y}_{\mathbf{o b s}}$ is the observed data, $\mathbf{Y}$ is data from the simulated data set, and $\hat{\mathbf{p}}_{0}(\Delta)$ is the maximum likelihood estimate of $\mathbf{p}_{0}$ given $\Delta$.

Suppose we simulate $B$ data sets from independent binomial distributions with the probabilities using $\widehat{\Delta}^{*}$ and $\widehat{\mathbf{p}}_{0}\left(\Delta^{*}\right)$ estimated from the observed data $\mathbf{y}_{\mathbf{o b s}}$. For studies with double zeros, although their estimated risk differences are zero, sample sizes from such studies are still valuable information in estimating the overall $\Delta$ and it confidence intervals [24]. Sample sizes from all studies including the ones with double zeros are used in the proposed method. The number of events are simulated from binomial distributions with the probabilities of $\widehat{\mathbf{p}}_{0}\left(\Delta^{*}\right)$.

The designated statistic of each simulated data set is computed, and compared with $T\left(\mathbf{y}_{\mathbf{o b s}}\right)$. The set of $T(\mathbf{Y}) \leq T\left(\mathbf{y}_{\mathbf{o b s}}\right)$ equals to $\Omega_{T}\left(\mathbf{y}_{\mathbf{o b s}}\right)=\{\mathbf{Y}: T(\mathbf{Y}) \leq$ $\left.T\left(\mathbf{y}_{\mathbf{o b s}}\right)\right\}$. Let the size of $\Omega_{T}\left(\mathbf{y}_{\mathbf{o b s}}\right)$ be $B_{1}$ with data: $\mathbf{Y}_{1}, \cdots$, $\mathbf{Y}_{B_{1}}$. Then, the upper limit in Eq. 3 can be rewritten as the supremum of $\Delta$ such that

$$
\widehat{G}(\Delta)=\frac{1}{B} \sum_{b=1}^{B_{1}} \frac{f\left(\mathbf{Y}_{\mathbf{b}} \mid \Delta, \widehat{\mathbf{p}}_{0}(\Delta)\right)}{f\left(\mathbf{Y}_{\mathbf{b}} \mid \widehat{\Delta}^{*}, \widehat{\mathbf{p}}_{0}\left(\Delta^{*}\right)\right)}>\frac{\alpha}{2},
$$

where $f\left(\mathbf{Y}_{\mathbf{b}}\right)$ is the probability density function of $\mathbf{Y}_{\mathbf{b}}$, which is a product of independent binomial distributions 
with parameters $\left(n_{i j}, p_{i j}\right)$ for the treatment $j$ in the $i$ th study. For a given $\Delta$, numerical algorithms can be used to find the maximum likelihood estimator of $\mathbf{p}_{0}(\Delta)$ to calculate $\widehat{G}(\Delta)$.

Similarly, the IS lower limit can be computed. It should be noted that designated statistics from the same model are used for the IS upper limit and the IS lower limit. For example, the asymptotic upper limit from the fixed-effects model is used as the designated statistic for the accurate upper limit, and then the lower limits from the same model is used for the accurate lower limit. We refer this accurate interval as the IS-F interval. When the asymptotic limits from the random-effects model are used as the designated statistics, the computed accurate limits are referred to be as the IS-R interval.

\section{Results}

We first use an example from 18 schizophrenia clinical trials to illustrate the application of the proposed accurate intervals. In addition to the $\mathrm{F}$ interval, the $\mathrm{R}$ interval, the IS-F interval, and the IS-R interval, We also include the confidence interval for $\Delta$ by Tian [9] in the comparison (referred to be as the Tian interval). Tian interval can be computed by using their developed $\mathrm{R}$ function meta.exact from the exactmeta function, without the mid-p value approach. All data including studies with zero events are used in the confidence interval calculation.

These 18 schizophrenia clinical trials reported the number of all-cause mortality for patients treated with the long-acting injectable antipsychotics (LAI-AP) or the oral antipsychotics (OAP) which is the control treatment here. Data of these 18 trials are presented in Table 2, which was provided by Efthimiou [25]. Out of a total of 3774 participants treated with the LAI-AP, 7 events were observed. In the OAP group, there were 6 events recorded from a total of 2145 participants in the control group. The naive estimates for all-cause mortality rates are $0.185 \%$ and $0.279 \%$ in the LAI-AP group and the OAP group, respectively.

Table 3 presents the estimated $\widehat{\Delta}$ and the $95 \%$ confidence interval for $\Delta$ using the five methods. The point estimate of $\widehat{\Delta}$ from the $\mathrm{R}$ method is similar to the Tian method, and they are larger than that from the F method. It can be seen that the Tian interval is much wider than others, and the asymptotic $\mathrm{F}$ or $\mathrm{R}$ intervals have shorter lengths than the proposed accurate intervals. The upper limits of the proposed accurate intervals are smaller than those of other intervals. All the intervals contain zero. Therefore, we fail to reject the null hypothesis that there is no difference between the LAI-AP treatment and the OAP treatment with regards to the all-cause mortality rate.

\section{Simulation studies}

We conduct extensive simulation studies to compare coverage probability and average length of the five intervals: the $\mathrm{F}$ interval, the $\mathrm{R}$ interval, the IS-F interval, the IS- $\mathrm{R}$ interval, and the Tian interval. The nominal confidence level is set as $95 \%$. The sample sizes, $n_{i j}$, are assumed to be the same as those in the aforementioned example, as $N_{1}$ and $N_{0}$ in Table 2. The number of responses $X_{i j 1}$ follows a binomial distribution $\left(n_{i j}, p_{i j}\right)$. We simulate $D=1,000$ data for each configuration: $\mathbf{Y}_{1}, \mathbf{Y}_{2}, \cdots$, and $\mathbf{Y}_{D}$. For the proposed IS intervals, we generate $B=2,000$ importance samples from the estimated distribution using each simulated data.

Coverage probability is defined as the proportion of the pre-specified risk difference $\Delta$ being included in the confidence intervals:

$$
C P=\frac{1}{D} \sum_{d=1}^{D} I\left(\Delta \in C I\left(\mathbf{Y}_{\mathbf{d}}\right)\right) .
$$

A confidence interval with the simulated interval being closer to the nominal level is preferable. Average length is defined as the average of all the lengths

$$
A L=\sum_{d=1}^{D} \frac{C I_{\text {upper }}\left(\mathbf{Y}_{\mathbf{d}}\right)-C I_{\text {lower }}\left(\mathbf{Y}_{\mathbf{d}}\right)}{D},
$$

where $C I_{\text {lower }}$ and $C I_{\text {upper }}$ are the lower limit and the upper limit of an interval. When two intervals are comparable with regards to coverage probability, the one with a shorter average length outperforms the other.

\section{Homogeneity of study effects}

We first compare the coverage probabilities of the five methods with fixed probabilities, $\mathbf{p}_{1}$ and $\mathbf{p}_{0}$. For simplicity, we assume a common rate in the control group, $p_{i 0}=p$, with $p$ from $0.01 \%$ to $10 \%$. The treatment probability is $p_{i 1}=p+\Delta$. For each configuration of $(p, \Delta)$, the coverage probabilities of these methods are computed, see Fig. 1 when $\Delta=0.005$ and 0.05 . It can be seen that the F method has the coverage closer to the nominal level when $\Delta=0.005$, except the case in which $p$ is very low. As $\Delta$ is increased to 0.05 , the F interval, the IS-R interval, and the IS-F interval have similar coverages when $p$ is small. The IS-F interval and the IS-R interval are conservative when $p$ is large. In this plot with $\Delta=0.05$, the Tian interval and the $\mathrm{R}$ interval have the coverage probabilities below the nominal level. Overall, the F interval has good performance with regards to coverage when studies are homogeneous and have common rates.

Given the number of nuisance probabilities, it is difficult to compare the performance of the five methods under each configuration. With 18 studies and 5 considered probabilities, the number of possible configurations is $5^{36}$, which is over $10^{25}$. For this reason, we follow the approach by Tian et al. [9] to compare the performance of these methods by simulating the probabilities of the control group $\left(\mathbf{p}_{0}\right)$ from uniform distributions: $U(0, b)$, where 
Table 2 Data from 18 clinical trials comparing all-cause mortality rate of patients treated with long-acting injectable antipsychotics (LAI-AP) or the oral antipsychotics (OAP) treatment as the control

\begin{tabular}{|c|c|c|c|c|c|c|}
\hline \multirow[b]{2}{*}{ Study } & \multicolumn{2}{|c|}{ LAI-AP group } & \multicolumn{2}{|c|}{ OAP group } & \multicolumn{2}{|c|}{ Sample sizes } \\
\hline & Events & Non-events & Events & Non-events & $N_{1}$ & $\mathrm{~N}_{2}$ \\
\hline Kane 2012 & 1 & 268 & 0 & 134 & 269 & 134 \\
\hline Kane 2014 & 0 & 168 & 0 & 172 & 168 & 172 \\
\hline Meltzer 2015 & 0 & 415 & 1 & 206 & 415 & 207 \\
\hline Hirsch 1973 & 0 & 41 & 0 & 40 & 41 & 40 \\
\hline Jolley 1990 & 2 & 25 & 0 & 27 & 27 & 27 \\
\hline Odejide 1952 & 0 & 35 & 1 & 34 & 35 & 35 \\
\hline Rifkin 1977 & 0 & 23 & 1 & 21 & 23 & 22 \\
\hline Lauriello 2008 & 0 & 306 & 0 & 98 & 306 & 98 \\
\hline Berwaerts 2015 & 0 & 160 & 0 & 145 & 160 & 145 \\
\hline Fu 2015 & 2 & 162 & 0 & 170 & 164 & 170 \\
\hline Gopal 2010 & 0 & 221 & 0 & 135 & 221 & 135 \\
\hline Hough 2010 & 0 & 206 & 0 & 204 & 206 & 204 \\
\hline Kramer 2010 & 0 & 163 & 0 & 84 & 163 & 84 \\
\hline Nasrallah 2010 & 1 & 390 & 1 & 126 & 391 & 127 \\
\hline Pandinda 2010 & 1 & 487 & 0 & 164 & 488 & 164 \\
\hline Takahasji 2013 & 0 & 160 & 1 & 163 & 160 & 164 \\
\hline Kane 2003 & 0 & 302 & 1 & 97 & 302 & 98 \\
\hline Nasser 2016 & 0 & 235 & 0 & 119 & 235 & 119 \\
\hline
\end{tabular}

$b=0.0001,0.001,0.01$, and 0.1 . We consider the following five $\Delta$ values: $0.001,0.005,0.01,0.05$, and 0.1 . Under the study homogeneity assumption, the probabilities of the treatment group $\mathbf{p}_{1}$ are then obtained as $p_{i 1}=p_{i 0}+\Delta$.

Table 4 presents coverage and average length comparison between the five intervals when $\mathbf{p}_{0} \sim U(0,0.01 \%)$. Coverage probabilities of the $\mathrm{F}$ interval range from $89 \%$ to $96 \%$. The $\mathrm{R}$ interval is very conservative when $\Delta$ is small, and its coverage is below $95 \%$ when $\Delta$ is larger. The Tian interval is conservative when $\Delta \leq 1 \%$, but it could be as low as $76 \%$ when $\Delta$ is $10 \%$. The proposed accurate intervals always have the coverage probabilities close to the nominal level as compared to the existing intervals. Average length is always an increasing function of $\Delta$ for each confidence interval method. The Tian intervals are wider than others when they all guarantee the coverage probability. The IS- $R$ interval generally has a shorter length as compared to the $\mathrm{R}$ interval and the IS-F intervals.
When the event rates of the control group are higher with $\mathbf{p}_{0} \sim U(0,0.1 \%)$ in Fig. 2 , the $\mathrm{F}$ interval generally performs better than others with regards to coverage probability and average length. When $\Delta$ is large (e.g., 10\%), coverage probabilities of these intervals are all slightly below $95 \%$. In this case with a small $\mathbf{p}_{0}$ and a relatively large $\Delta$, the proposed intervals (IS-R or IS-F intervals) have better coverage probabilities than the $\mathrm{F}$ interval, and the length difference between the accurate intervals and the F interval is small. When $\Delta=10 \%$, the coverage probability of the Tian interval is below $80 \%$. When the rates are even higher with $\mathbf{p}_{0} \sim U(0,1 \%)$, the rates are not rare in these configurations, and the $\mathrm{F}$ interval outperforms others as seen in Fig. 2.

\section{Heterogeneity of study effects}

Under the study heterogeneity assumption, the probability in the treatment group is $p_{i 1}=p_{i 0}+u_{i}$, where $u_{i}$ is

Table 3 Confidence intervals for risk difference between the LAI-AP group and the OAP group

\begin{tabular}{|c|c|c|c|c|c|c|c|c|c|c|}
\hline \multirow[b]{2}{*}{ Method } & \multirow[b]{2}{*}{$\widehat{\Delta}$} & \multicolumn{3}{|c|}{ Asymptotic intervals } & \multicolumn{3}{|c|}{ IS intervals } & \multicolumn{3}{|c|}{ Tian interval } \\
\hline & & lower & upper & length & lower & upper & length & lower & upper & length \\
\hline Fixed-effects & $-0.064 \%$ & $-0.346 \%$ & $0.218 \%$ & $0.564 \%$ & $-0.506 \%$ & $0.165 \%$ & $0.704 \%$ & & & \\
\hline Random-effects & $-0.030 \%$ & $-0.382 \%$ & $0.322 \%$ & $0.671 \%$ & $-0.509 \%$ & $0.250 \%$ & $0.758 \%$ & & & \\
\hline Tian method & $-0.028 \%$ & & & & & & & $-0.843 \%$ & $0.430 \%$ & 1.273 \\
\hline
\end{tabular}




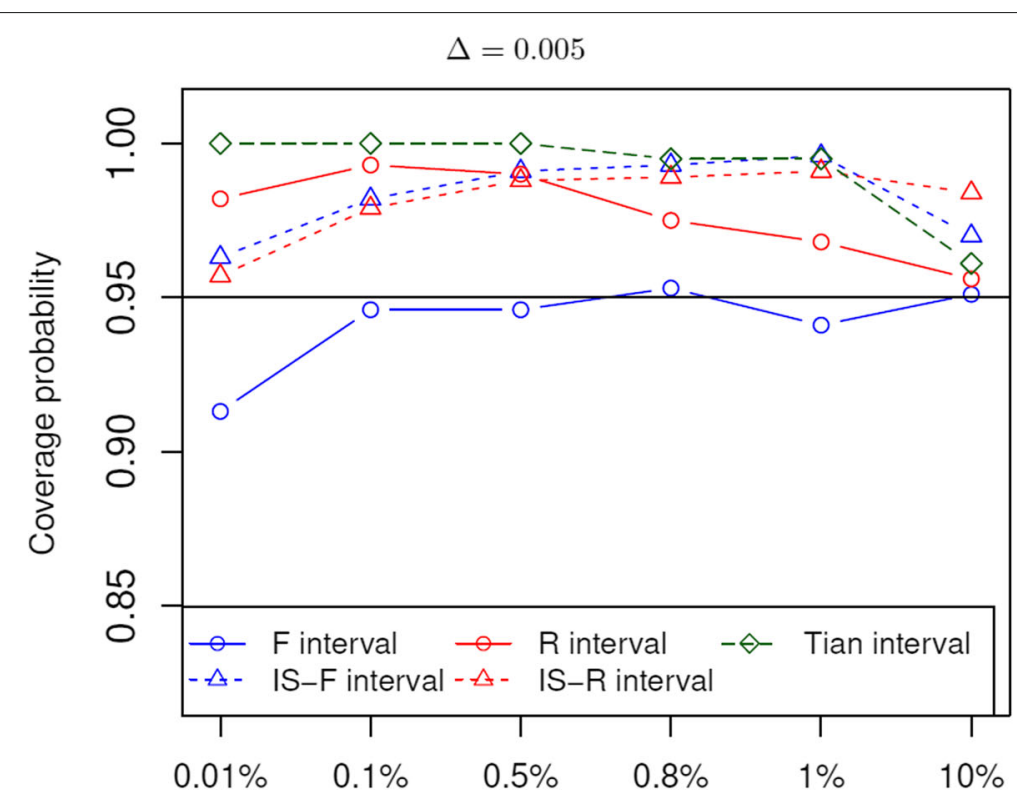

p

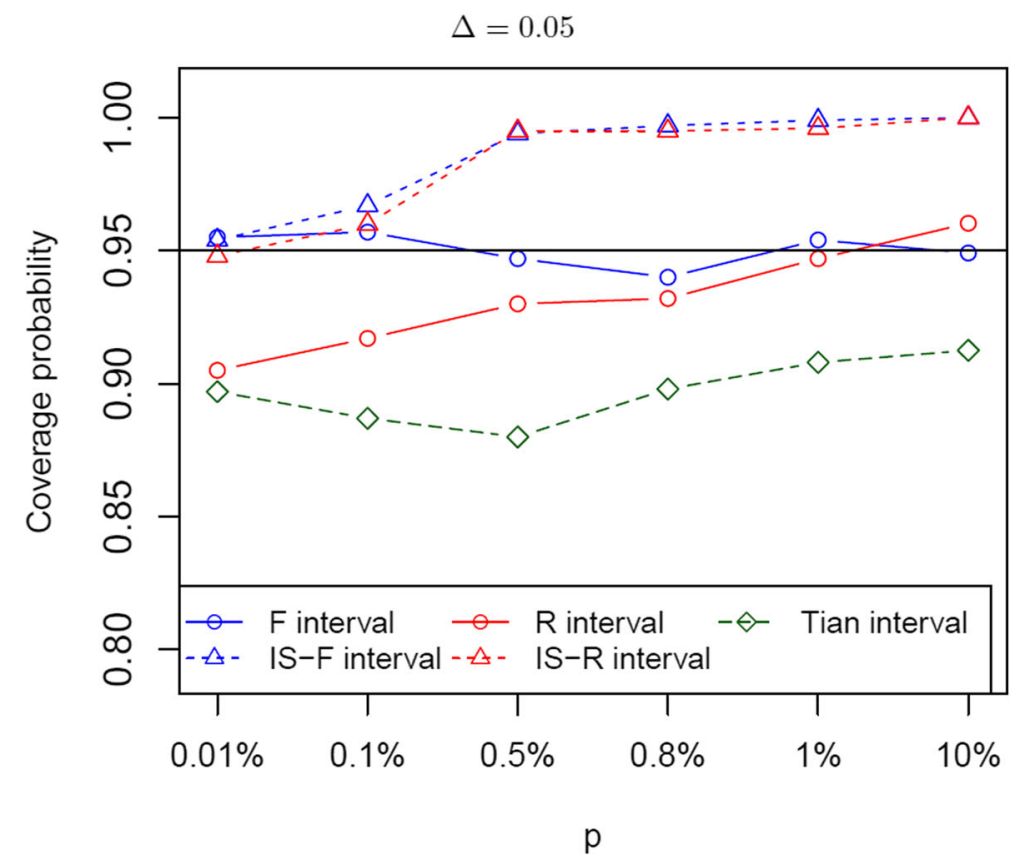

Fig. 1 Coverage probability of the five methods under the study homogeneity assumption, with fixed and common rate $p_{i 0}=p$ in the control group

Table 4 Coverage probability and average length comparison between the five intervals when $\mathbf{p}_{0} \sim U(0,0.01 \%)$

\begin{tabular}{|c|c|c|c|c|c|c|c|c|c|c|}
\hline \multirow[b]{2}{*}{$\underline{\Delta}$} & \multicolumn{2}{|c|}{ The F interval } & \multicolumn{2}{|c|}{ The IS-F interval } & \multicolumn{2}{|c|}{ The R interval } & \multicolumn{2}{|c|}{ The IS-R interval } & \multicolumn{2}{|c|}{ The Tian interval } \\
\hline & Coverage & Length & Coverage & Length & Coverage & Length & Coverage & Length & Coverage & Length \\
\hline $0.1 \%$ & 0.890 & $0.21 \%$ & 0.968 & $0.36 \%$ & 1.000 & $0.63 \%$ & 0.984 & $0.34 \%$ & 0.987 & $1.02 \%$ \\
\hline $0.5 \%$ & 0.932 & $0.47 \%$ & 0.970 & $0.71 \%$ & 0.987 & $0.72 \%$ & 0.957 & $0.67 \%$ & 0.997 & $1.10 \%$ \\
\hline $1.0 \%$ & 0.949 & $0.66 \%$ & 0.958 & $0.83 \%$ & 0.877 & $0.84 \%$ & 0.944 & $0.76 \%$ & 0.994 & $1.24 \%$ \\
\hline $5.0 \%$ & 0.949 & $1.45 \%$ & 0.946 & $1.45 \%$ & 0.905 & $1.58 \%$ & 0.952 & $1.46 \%$ & 0.903 & $1.52 \%$ \\
\hline $10.0 \%$ & 0.962 & $1.99 \%$ & 0.962 & $2.00 \%$ & 0.935 & $2.15 \%$ & 0.960 & $2.00 \%$ & 0.730 & $1.63 \%$ \\
\hline
\end{tabular}




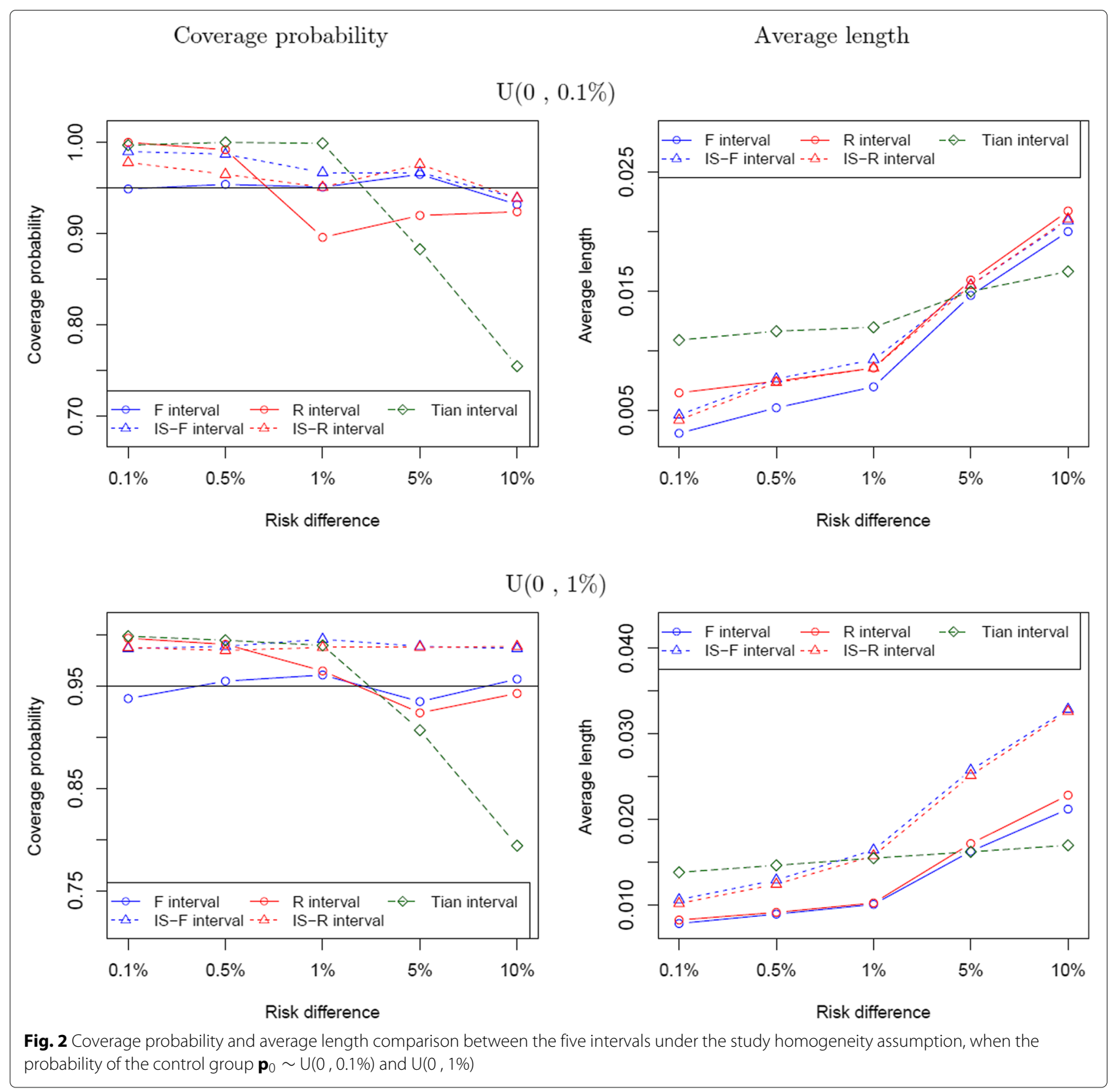

the random study effect that follows a normal distribution with mean of $\Delta$ and standard deviation of $\Delta / 2$. Figure 3 presents the coverage probability and average length comparison between the five intervals when $\mathbf{p}_{0} \sim U(0,0.01 \%)$, $U(0,0.1 \%)$, and $U(0,1 \%)$. As $\Delta$ increases, the standard deviation of the probabilities in the treatment group goes up. When $\Delta$ is small, the $\mathrm{F}$ interval, the IS- $\mathrm{R}$ interval, and the IS-F interval have the coverage probabilities closer to the nominal level as compared to the $\mathrm{R}$ interval and the Tian interval. Coverage probabilities of the $\mathrm{F}$ interval and the IS-F interval drop to almost $50 \%$ when $\Delta$ is
$10 \%$. The $\mathrm{R}$ interval generally has good coverage when $\Delta$ is large. However, the $\mathrm{R}$ interval's coverage probabilities are very low when $\Delta=1 \%$ in meta-analysis with rare events (e.g., $\mathbf{p}_{0} \sim U(0,0.01 \%)$ or $\left.U(0,0.1 \%)\right)$. The IS$\mathrm{R}$ interval has consistent good performance with regards to coverage and length as compared to others in metaanalysis with rare events. Figure 3 also presents the results when the event rates are not rare (e.g., $\mathbf{p}_{0} \sim U(0,1 \%)$ ). When $\Delta$ is large, the $\mathrm{R}$ interval and the IS- $\mathrm{R}$ interval have better coverage probabilities than others. When variance of study effects is small (for the configurations with 


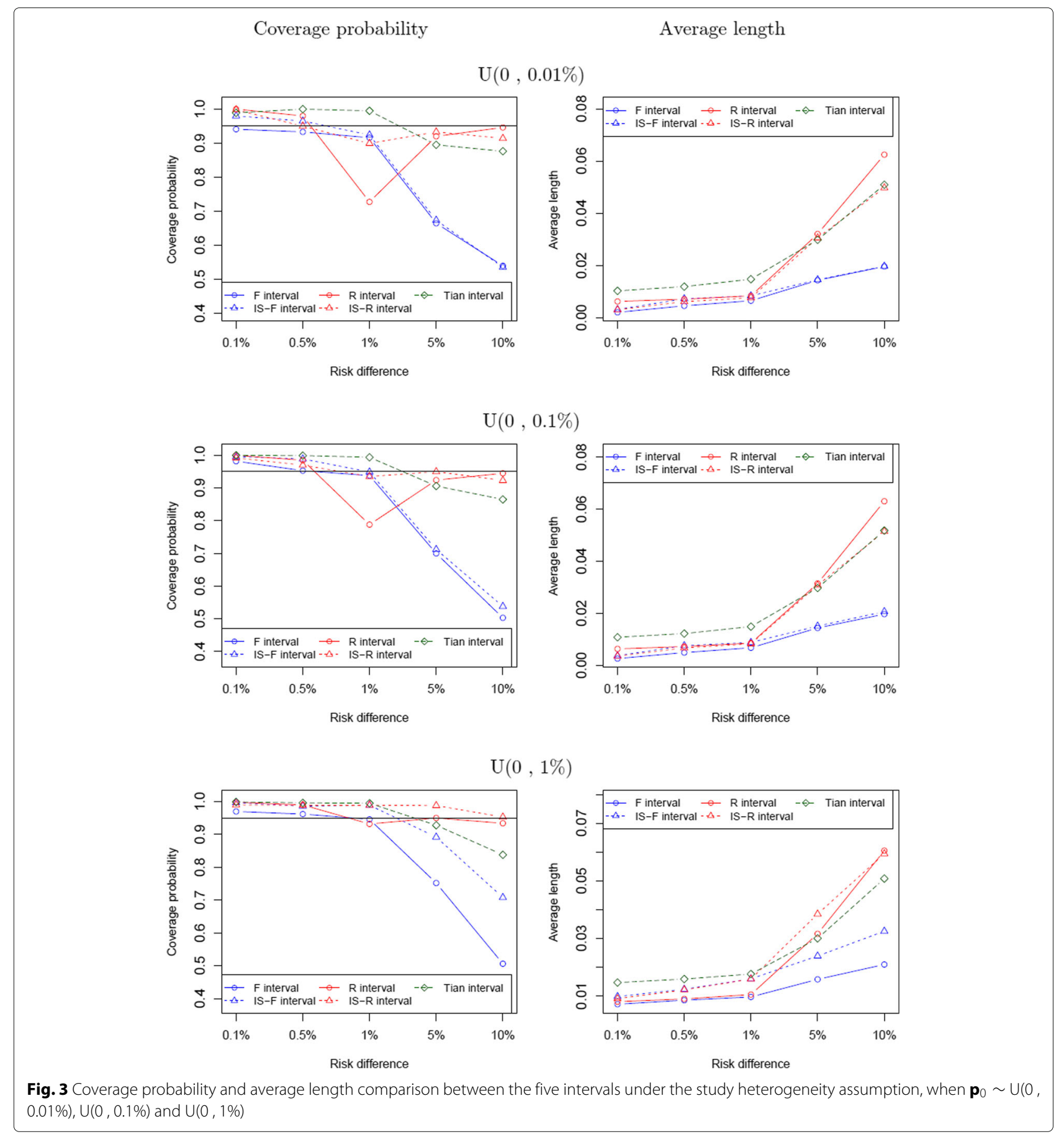

small $\Delta$ values), the $\mathrm{F}$ interval performs better where the configurations are similar to the ones under the study homogeneity assumption.

\section{Conclusions}

We propose using importance sampling to construct confidence intervals for risk difference in meta-analysis with rare events. The traditionally used $\mathrm{F}$ interval has satisfactory performance with regards to coverage probability and interval length when the rate of events is not rare under the study homogeneity assumption, but this interval could have a very low coverage probability under the study heterogeneity assumption. The IS-R interval based on the asymptotic limits from the random-effects model outperforms the existing intervals under the heterogeneity assumption. The IS intervals use the existing 
asymptotic limits to order the sample space. Although the asymptotic limits are computed from asymptotic approaches whose performances are based on the approximation of the test statistic to the limiting distribution, the order of these limits provides a useful information to produce better IS limits.

The Tian interval often guarantees the coverage probability when the rates of both groups are rare, but that interval could have the coverage probability below the nominal level when $\Delta$ is large. Theoretically, the Tian interval can be used as a designated statistic to order the sample space. However, simulations are involved in the Tian interval calculation that would significantly increase the computational intensity of the proposed IS intervals. In addition, the ordering of the sample space based on the Tian interval may change as the number of simulations being utilized. For these reasons, we do not include the IS intervals based on the ordering by the Tian interval.

\section{Discussion}

The method by Buehler [13] to construct exact one-sided confidence interval is ideal for binary outcome when the size of the sample size is not too large that allows a full enumeration of the sample space [16, 26-29]. However, it is not feasible in meta-analysis as it is extremely difficult to save the sample space under the unconditional framework with sample size in each treatment group fixed. If the upper bound of the possible number of events can be determined and the size of the sample size is not too large, exact Buehler interval may be computed. Otherwise, an efficient search algorithm should be developed to order the sample space efficiently.

Exact confidence intervals are preferable for statistical inference. However, it is often computationally intensive, such as the aforementioned the exact interval by Buehler $[28,30-32]$. For these reasons, simulation based intervals are proposed for use in practice, including the proposed interval here, the Tian interval, and the interval based on confidence distribution [24, 33-35]. It is still a big challenge in exact meta-analysis by enumerating all possible data, which becomes a big data problem with the requirement of huge memory and computational power.

In addition to risk difference, odd ratio and risk ratio are also used to measure the treatment effect. For studies with zero events in one or both treatment groups, the estimated risk difference is zero. However, the estimated ratios could be infinity [17, 36-39]. In order to avoid this issue, an arbitrary small number (e.g., $\epsilon=0.5,1$ ) is often added to each cell in the data. The performance of the test statistics is affected by the chosen small value [6, 40-42]. The added value $\epsilon$ also raises the question of whether the number of participants in a study should be $n_{i j}$ or $n_{i j}+2 \epsilon$. We consider this as future work to study the IS intervals for ratios.

\section{Abbreviations}

GLMMS: Generalized linear mixed models; IS: Importance sampling; LAI-AP:

Long-acting injectable antipsychotics; OAP: Oral antipsychotics

\section{Acknowledgements}

We would like to thank the support from the supercomputing center at UNLV.

\section{Authors' contributions}

The idea for the paper was originally developed by GS. GS computed the new confidence interval for meta-analysis with rare binary outcome. GS, CB, and TJ drafted the manuscript and approved the final version.

\section{Funding}

Shan's research is partially supported by grants from the National Institute of General Medical Sciences from the National Institutes of Health:

P20GM109025. Jiang's work is supported by the National Natural Foundation of China under grant 11971433, and the First Class Discipline of Zhejiang -A

(Zhejiang Gongshang University-Statistics).

\section{Availability of data and materials}

Not applicable. This is a manuscript to develop novel statistical approaches, therefore, no real data is involved.

Ethics approval and consent to participate Not applicable.

Consent for publication

Not applicable.

\section{Competing interests}

The authors declare that they have no competing interests.

\section{Author details}

${ }^{1}$ School of Statistics and Mathematics, and School of Business, Zhejiang Gongshang University, Hangzhou, Zhejiang, China. ${ }^{2}$ School of Mathematical Sciences, Nankai University, Tianjin, China. ${ }^{3}$ Epidemiology and Biostatistics Program, School of Public Health, University of Nevada Las Vegas, Las Vegas, USA.

Received: 26 September 2019 Accepted: 17 March 2020

Published online: 30 April 2020

\section{References}

1. Vandermeer B, Bialy L, Hooton N, Hartling L, Klassen TP, Johnston BC Wiebe N. Meta-analyses of safety data: a comparison of exact versus asymptotic methods. Stat Methods Med Res. 2009;18(4):421-32. https:// doi.org/10.1177/0962280208092559.

2. Mehta CR, Patel NR, Gray R. Computing an Exact Confidence Interval for the Common Odds Ratio in Several 2 * 2 Contingency Tables. J Am Stat Assoc. 1985;80(392):969-73. https://doi.org/10.1080/01621459.1985. 10478212.

3. Cai T, Parast L, Ryan L. Meta-analysis for rare events. Stat Med. 2010;29(20):2078-89. https://doi.org/10.1002/sim.3964.

4. Wan X, Wang W, Liu J, Tong T. Estimating the sample mean and standard deviation from the sample size, median, range and/or interquartile range. BMC Med Res Methodol. 2014;14(1):135. https://doi. org/10.1186/1471-2288-14-135

5. Mantel N, Haenszel W. Statistical Aspects of the Analysis of Data From Retrospective Studies of Disease. JNCI J Natl Cancer Inst. 1959;22(4): 719-48. https://doi.org/10.1093/jnci/22.4.719.

6. Sweeting MJ, Sutton AJ, Lambert PC. What to add to nothing? Use and avoidance of continuity corrections in meta-analysis of sparse data. Stat Med. 2004;23(9):1351-75. https://doi.org/10.1002/sim.1761.

7. Rücker G, Schwarzer G, Carpenter J, Olkin I. Why add anything to nothing? The arcsine difference as a measure of treatment effect in meta-analysis with zero cells. Stat Med. 2009;28(5):721-38. https://doi. org/10.1002/sim.3511.

8. Kuss $\mathrm{O}$. Statistical methods for meta-analyses including information from studies without any events-add nothing to nothing and succeed nevertheless. Stat Med. 2015;34(7):1097-116. https://doi.org/10.1002/sim 6383. 
9. Tian L, Cai T, Pfeffer MA, Piankov N, Cremieux P-Y, Wei L. Exact and efficient inference procedure for meta-analysis and its application to the analysis of independent $2 \times 2$ tables with all available data but without artificial continuity correction. Biostat (Oxford Engl). 2009;10(2):275-81. https://doi.org/10.1093/biostatistics/kxn034.

10. DerSimonian R, Laird N. Meta-analysis in clinical trials. Control Clin Trials. 1986;7(3):177-88.

11. Schwarzer G, Carpenter JR, Rücker G. Meta-Analysis with R, Use R! Cham: Springer; 2015. https://doi.org/10.1007/978-3-319-21416-0. http://link. springer.com/10.1007/978-3-319-21416-0.

12. Bakbergenuly I, Kulinskaya E. Meta-analysis of binary outcomes via generalized linear mixed models: A simulation study. BMC Med Res Methodol. 2018;18(1):70. https://doi.org/10.1186/s12874-018-0531-9.

13. Buehler RJ. Confidence intervals for the product of two binomial parameters. J Am Stat Assoc. 1957;52(280):482-93.

14. Kabaila P, Lloyd CJ. The efficiency of Buehler confidence limits. Stat Probab Lett. 2003;65(1):21-8. https://doi.org/10.1016/s01677152(03)00215-3.

15. Kabaila P, Lloyd CJ. Buehler confidence limits and nesting. Aust N Z J Stat. 2004;46(3):463-9. https://doi.org/10.1111/j.1467-842x.2004.00343.x.

16. Kabaila P. Computation of exact confidence limits from discrete data. Comput Stat. 2005;20(3):401-14. https://doi.org/10.1007/bf02741305.

17. Shan G. Exact Statistical Inference for Categorical Data, 1st edn. San Diego: Academic Press; 2015. http://www.worldcat.org/isbn/0081006810.

18. Lloyd CJ, Li D. Computing highly accurate confidence limits from discrete data using importance sampling. Stat Comput. 2014;24(4):663-73. https://doi.org/10.1007/s11222-013-9409-1.

19. Kabaila P, Lloyd CJ. Profile upper Confidence Limits from Discrete Data. Aust N Z J Stat. 2000;42(1):67-79. https://doi.org/10.1111/1467-842X. 00108.

20. Garthwaite PH, Buckland ST. Generating Monte Carlo confidence intervals by the Robbins- Monro process. J Comput Graph Stat. 1992;41(1):159-71.

21. Garthwaite PH, Jones MC. A stochastic approximation method and its application to confidence intervals. Journal of Computational and Graphical Statistics. 2009;18(1):184-200.

22. Viechtbauer W. Conducting Meta-Analyses in $<i>R</ i>$ with the $<$ b $>$ metafor</b> Package. J Stat Softw. 2010;36(3):1-48. https://doi.org/ 10.18637/jss.v036.i03.

23. Lloyd CJ. Accurate confidence limits for stratified clinical trials. Stat Med. 2013;32(20):3415-23. https://doi.org/10.1002/sim.5809.

24. Yang G, Liu D, Wang J, Xie MG. Meta-analysis framework for exact inferences with application to the analysis of rare events. Biometrics. 2016;72(4):1378-86. https://doi.org/10.1111/biom.12497.

25. Efthimiou O. Practical guide to the meta-analysis of rare events. Evid Based Ment Health. 2018:21(2):72-6. https://doi.org/10.1136/eb-2018-102911.

26. Kabaila P, Lloyd CJ. Tight upper confidence limits from discrete data. Aust J Stat. 1997;39(2):193-204. https://doi.org/10.1111/j.1467-842X.1997. tb00535.x.

27. Kabaila Paul. Better Buehler confidence limits. Stat Probab Lett. 2001;52(2):145-54.

28. Shan G, Banks S, Miller JB, Ritter A, Bernick C, Lombardo J, Cummings JL. Statistical advances in clinical trials and clinical research. Alzheimers Dement Transl Res Clin Interv. 2018;4:366-71.

29. Shan $\mathrm{G}$. Exact confidence limits for the probability of response in two-stage designs. Statistics. 2018;52(5):1086-95. https://doi.org/10. 1080/02331888.2018.1469023

30. Shan G. Exact Tests for Disease Prevalence Studies With Partially Validated Data. Stat Biopharm Res. 20191-14. https://doi.org/10.1080/19466315. 2018.1555099.

31. Shan G. Exact confidence limits for the response rate in two-stage designs with over or under enrollment in the second stage. Stat Methods Med Res. 2018;27(4):1045-55.

32. Zhang H, Shan G. Letter to Editor: A novel confidence interval for a single proportion in the presence of clustered binary outcome data. Stat Methods Med Res. 2019096228021984005. https://doi.org/10.1177/ 0962280219840056.

33. Liu D, Liu RY, ge Xie M. Exact Meta-Analysis Approach for Discrete Data and its Application to 22 Tables With Rare Events. J Am Stat Assoc. 2014;109(508):1450-65. https://doi.org/10.1080/01621459.2014.946318.
34. Shan G, Ma C, Hutson AD, Wilding GE. Randomized Two-Stage Phase II Clinical Trial Designs Based on Barnard's Exact Test. J Biopharm Stat. 2013;23(5):1081-90. https://doi.org/10.1080/10543406.2013.813525.

35. Shan $G$, Zhang $H$, Jiang $T$. Minimax and admissible adaptive two-stage designs in phase II clinical trials. BMC Med Res Methodol. 2016;16(1):90. https://doi.org/10.1186/s12874-016-0194-3.

36. Shan G, Hutson AD, Wilding GE. Two-stage k-sample designs for the ordered alternative problem. Pharm Stat. 2012;11(4):287-94. https://doi. org/10.1002/pst.1499.

37. Shan $G, M a C$, Hutson AD, Wilding GE. Some tests for detecting trends based on the modified Baumgartner-Weiß-Schindler statistics. Comput Stat Data Anal. 2013;57(1):246-61. https://doi.org/10.1016/j.csda.2012.04. 021.

38. Shan G, Wilding GE. Powerful Exact Unconditional Tests for Agreement between Two Raters with Binary Endpoints. PLoS ONE. 2014;9(5):97386. https://doi.org/10.1371/journal.pone.0097386.

39. Shan G, Wilding GE, Hutson AD, Gerstenberger S. Optimal adaptive two-stage designs for early phase II clinical trials. Stat Med. 2016;35(8): 1257-66. https://doi.org/10.1002/sim.6794.

40. Shan G, Kang L, Xiao M, Zhang H, Jiang T. Accurate unconditional p-values for a two-arm study with binary endpoints. J Stat Comput Simul. 2018;88(6):1200-10.

41. Shan G. Comments on 'Two-sample binary phase 2 trials with low type error and low sample size'. Stat Med. 2017;36(21):3437-8. https://doi.org/ 10.1002/sim.7359.

42. Shan G, Gerstenberger S. Fisher's exact approach for post hoc analysis of a chi-squared test. PLoS ONE. 2017;12(12):0188709. https://doi.org/10. 1371/journal.pone.0188709.

\section{Publisher's Note}

Springer Nature remains neutral with regard to jurisdictional claims in published maps and institutional affiliations.

\section{Ready to submit your research? Choose BMC and benefit from:}

- fast, convenient online submission

- thorough peer review by experienced researchers in your field

- rapid publication on acceptance

- support for research data, including large and complex data types

- gold Open Access which fosters wider collaboration and increased citations

- maximum visibility for your research: over $100 \mathrm{M}$ website views per yea

At $B M C$, research is always in progress.

Learn more biomedcentral.com/submissions 